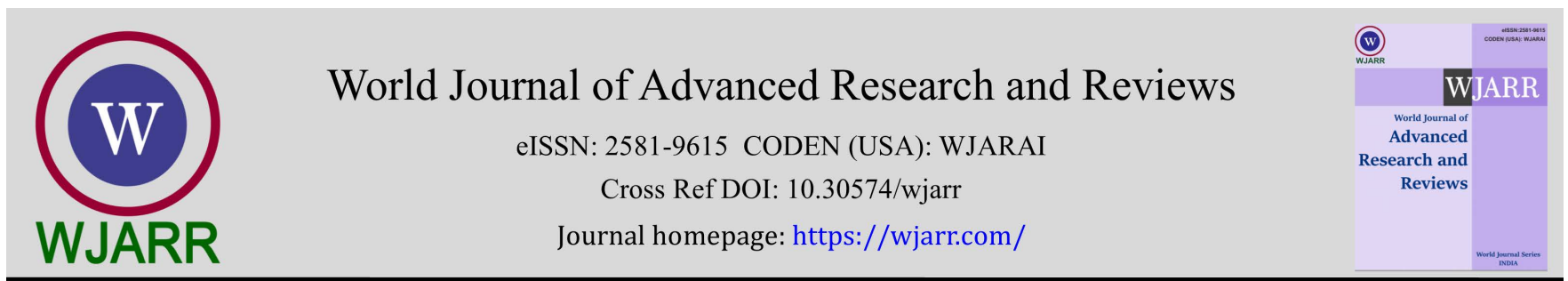

(RESEARCH ARTicle)

Check for updates

\title{
Morphological variation of iron toxicity tolerance in lowland rice (Oryza sativa L.) varieties
}

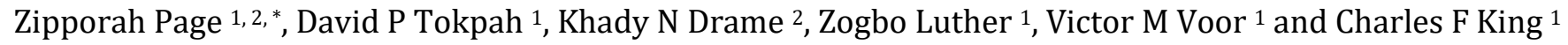 \\ ${ }^{1}$ Central Agricultural Research Institute, Suakoko, Bong County, Liberia. \\ 2 Department of Crop and Horticulture Sokoine of Agriculture Morogoro, Tanzania.
}

World Journal of Advanced Research and Reviews, 2022, 13(01), 038-046

Publication history: Received on 29 November 2021; revised on 01 January 2022; accepted on 03 January 2022

Article DOI: https://doi.org/10.30574/wjarr.2022.13.1.0758

\begin{abstract}
Rice (Oryza sativa L.) is a staple food crop in many countries in Africa. Africa consumes 11.6 million tons of rice per annum and out of 39 rice-producing countries, 21 import $50 \%$ to $99 \%$ of their rice requirements. The inability to reach the yield potential that would sustain Africa's need for rice is due to many biotic and abiotic constraints that rice production faces. In lowland grown rice, one of the abiotic factors hindering rice production is iron toxicity. Excess uptake of ferrous (Fe2+) ions leads to a physiological stress, which results, into poor production. The current study aimed at selection of varieties tolerant to iron toxicity and assessment of the genetic diversity linked to this trait. In a hydroponic experiment conducted in a screen house at Africa Rice Centre in Dar es Salaam, 32 rice varieties were evaluated for tolerance to iron toxicity. The experiment was laid out in a split plot design with iron concentration as the main plot factor and variety as the sub plot factor. Two levels of iron concentration were used: 2 ppm and 300 ppm of $\mathrm{Fe} 2+$ as control and test concentrations, respectively. Traits observed to gauge tolerance were leaf bronzing (an indicator of iron toxicity), plant height, tillering, number of leaves, shoot weight (above ground), root length and root weight. The varieties ARICA8, and CK801 were found to be tolerant due to low bronzing indices, higher shoot weight, more number of leaves and lack of significant variation in morphology between the two Fe treatments except for the plant height. Correlation analysis depicted negative correlation between leaf bronzing and the other traits measured especially shoot biomass.
\end{abstract}

Keywords: Iron Toxicity; Tolerance; Genetic Variation; Bronzing

\section{Introduction}

Rice (Oryza sativa $L$ ) is the worlds' most important food crop, serving as staple food for more than half of the world population (Gaikwad et al., 2014). It belongs to the family of gramineae and supplies $20 \%$ of the calories consumed by humans. Lowland rice is cultivated on approximately 128 million hectares of irrigated and rain-fed land (Khadka et al., 2013). As many as 100 million hectares show some sort of nutritional constraints to rice growth caused by either deficiencies or toxicities (Keaton et al., 2017). Ferrous iron toxicity is among the constraints and primarily affects lowland rice grown on acid flooded soils that are rich in reducible iron (Bongoua et al., 2013). Increasing significant occurrence of iron toxicity make it a serious long-term threat to lowland rice production. Large areas of wetland ideally suited for rice production remain underused, especially in Asia, South America, West and Central Africa, because of iron toxicity stress (Sikirou et al., 2015).

In West Africa, iron toxicity is widespread throughout the humid forest and Savanna zones in about 30 to $40 \%$ of the cultivated lowlands (Aschet al 2005). Rice yield losses due to iron toxicity are reportedly ranging from 12 to $100 \%$, depending on the severity of the toxicity and the tolerance of the rice cultivars (Venus et al., 2013).

\footnotetext{
${ }^{*}$ Corresponding author: Zipporah Page

Central Agricultural Research Institute, Suakoko, Bong County, Liberia.

Copyright $(2022$ Author(s) retain the copyright of this article. This article is published under the terms of the Creative Commons Attribution Liscense 4.0.
} 
In Liberia, iron toxicity manifestation is mainly a result of heavy rainfalls and river over flows into the valleys. Stagnant inundation of the iron rich soils ultimately lead to the accumulation of high levels of $\mathrm{Fe}^{2+}$ in the soil solution. Rice grown in such environments takes up large amounts of ferrous iron, exceeding plant growth's requirements. As a result, plant metabolism is disturbed and rice yield is dramatically reduced. Data on iron toxicity distribution in Liberia is scanty. According to farmers, "lowlands are abandoned and remain uncultivated due to iron toxicity syndrome". Regarding how widespread the constraint was, the government instituted several management options to minimize iron toxicity in the rice fields such as application of limestone fertilizers, ridges, direct planting, and flushing the plot with fresh water but with little success.

\section{Material and methods}

\subsection{Plant Materials and Location of Study}

A hydroponic culture screening experiment was conducted in a screen house at the Africa Rice Centre in Mikocheni, Dares Salaam, and Tanzania. Exact geographical coordinates are as follows: latitude $6^{\circ} 45^{\prime} 49.4748^{\prime \prime}$ S, longitude $39^{\circ} 14^{\prime}$ 43.2312" E and altitude $17 \mathrm{~m}$ above sea level. The experiment was conducted from December 2014 to April 2015 followed by lab work at the same location. Thirty-two rice varieties, including tolerant and susceptible checks were screened for tolerance to iron toxicity. The tolerant checks were Suakoko - 8 and Wita- 4, WITA-4, while the susceptible check was IR64.

\subsection{Selection of Tolerant Varieties and Experimental setup}

To distinguish rice varieties tolerant to iron toxicity from the susceptible ones, the seedlings were treated with toxic levels of iron in a hydroponic experiment as described by (Priya et al., 2013). Seeds were sown into a perforated polystyrene plate floating on the surface of nutrient solution contained in 14 L-plastic trays. For each variety, 6 plants were grown at a rate of two plants per hole. Yoshida standard rice nutrient solution was used as the normal treatment for plant growth. For $\mathrm{Fe}^{2+}$ stress treatment, 300 ppm Fe ${ }^{2+}$ was added as $\mathrm{FeSO}_{4}$ to the standard nutrient solution. To maintain acidic conditions, the $\mathrm{pH}$ of the solution was adjusted once a week at 5.6-5-7 using $1 \mathrm{~N} \mathrm{NaOH}$ or $1 \mathrm{~N} \mathrm{HCl}$ at the time of solution change (every week). Fe stress treatment was maintained for3weeks.

\subsection{Experimental design}

The experiment was conducted in a randomized complete block design arranged in a split plot manner with four replications. The main plot had two levels of iron concentration and thirty two (32) varieties were allocated to the subplots. Each iron concentration (main plot) was assigned to one tray $(28 \mathrm{~cm} \mathrm{x} 31 \mathrm{~cm})$ containing nutrient solution with 2 or $300 \mathrm{ppm} \mathrm{Fe}^{2+}$. All 32 varieties were planted on the same polystyrene float $(26 \mathrm{~cm} \times 29 \mathrm{~cm})$ with a spacing of $1.5 \mathrm{~cm}$ $\mathrm{x} 1.5 \mathrm{~cm}$ between plants and between varieties. Each variety (sub-plot) occupied 3 holes on the float.

\subsection{Environmental Conditions}

The experiment was conducted in a screen house at ambient conditions at Africa Rice Centre in Mikocheni. Dar es Salaam, Tanzania. The temperature and relative humidity were recorded using a data logger (Dickson Data Logger TP125) between December 2014 and April 2015. The variables collected in the experiment were leaf bronzing, plant height, number of leaves, number tillers, dry shoot weight, root length dry and root weight.

\subsection{Scoring of Leaf Bronzing}

The leaf bronzing score was recorded every week starting from the first observation (10 days after imposing the stress) in reference to the scale of the Standard Evaluation system for Rice (IRRI, 2002). A scale varies from score 0 to 9 was set up by our lab and was adopted for iron toxicity severity.

\subsection{Number of tillers, number of leaves, and root length}

Number of tillers and leaves were collected by counting on each plant and data were recorded. Plant height was measured using a steel ruler from the ground level to the tip of the longest leaf. Root length was measured using the same steel ruler. 


\subsection{Dry weight of shoot and root}

Six plants per variety were harvested fresh at the end of the stress period, at 5 weeks after sowing to determine dry matter accumulation. Separate root and shoot samples were wrapped in an aluminum foil and oven dried at $72^{\circ} \mathrm{C}$ to constant weight. Then dry weights were determined. To determine trait variation between Fe stress and control, relative performances were calculated follows:

Relative reduction $=\frac{\mathrm{DW}_{\text {control plant }}-\mathrm{DW}_{\text {treated plant }}}{\mathrm{DW}_{\text {control plant }}} \times 100$

This was calculated for all the parameters except leaf bronzing which was observed only on Fe-treated plants.

\subsection{Data analysis}

Data analysis was done using Genstat statistical package (15th edition 2015). ANOVA was performed to analyze the experimental data on plant height, number of tillers and leaves, shoot weight, root weight, root length and leaf bronzing. Means separation was done using Duncan Multiple Range test. Correlation analysis was further performed to establish relationship between leaf bronzing and other traits (plant height, tillers, leaves, shoot weight, root weight, root length).

\section{Results}

\subsection{Environmental Conditions during the Experiment}

The hydroponic culture experiment was conducted in a screen house for five weeks including three weeks of stress to validate and select varieties, which are tolerant to iron toxicity. Environmental conditions during this period indicated that daily maximum temperature ranged from $45.60 \mathrm{C}$ to $49.10 \mathrm{C}$ while the minimum daily temperature was between $22.9^{\circ} \mathrm{C}$ and $32.5^{\circ} \mathrm{C}$. The highest temperature was recorded in March while the minimum temperature was in December 2014 (Fig. 1). The average minimum and maximum temperature during the growing season were $34.4^{\circ} \mathrm{C}$ and $39.4^{\circ} \mathrm{C}$, which was favorable for rice production. The maximum daily relative humidity (RH) ranged from $89.7 \%$ to $99.5 \%$ while the minimum daily relative humidity ranged from $26.6 \%$ to $28.5 \%$. The highest and lowest relative humidity were both observed in March suggesting large variations of RH. The average minimum and maximum relative humidity during the experimental period was $61.3 \%$ and $77.9 \%$, which was favorable for rice production described in (Fig. 1). Plants, the more intense the effect on plant growth was. Thus at week three, leaf bronzing increased and many varieties stopped growing.

\subsection{Effects of Fe2+ stress on the varieties tested}

The progresses of Fe2+ stress on the varieties tested at different stages were shown at week one, the reduction of plant growth was visible on leaf number and leaf width. At week two, leaf-bronzing symptoms appeared on Fe-treated plants, plant height, and shoot biomass were strongly reduced. The longer the duration of Fe2+ treatment on the plants, the more intense the effect on plant growth was. Thus at week three, leaf bronzing increased and many varieties stopped growing. Plants, the more intense the effect on plant growth was. Thus at week three, leaf bronzing increased and many varieties stopped growing.

\subsection{Variation of plant height, leaf number, tiller number and root length among the varieties and Fe treatments}

After three weeks of exposure to Fe stress (300 ppm Fe2+), plant height of tested varieties for the control treatment ranged from $47.5 \mathrm{~cm}$ (Saro 5) to $90.5 \mathrm{~cm}$ (Shaka102) with a mean of $68.6 \mathrm{~cm}$ while for the $\mathrm{Fe} 2+$ treatment, average plant height was $48.9 \mathrm{~cm}$ with the highest height attained by CK $90(68.67 \mathrm{~cm})$ and the lowest by Saro5 $(34.2 \mathrm{~cm})$. The range of leaf number under control treatment was between 6.5 (CK 73) and 12.6 (IR 64) with a mean of 9.9 while for Fe treatment the range was between 4.5 (Botry) and 7.3 (Shaka 102) with a mean of 6.1. Tiller number for control plants ranged from 0.0 (CK 73) to 1.9 (Orylux4) with a mean of 0.8 while under $\mathrm{Fe} 2+$ treatment, the range was from 0.0 (IR64) to 0.5 (Jasmine 85) with a mean of 0.1. Root length under control treatment ranged from $15 \mathrm{~cm}$ (Jasmine 85) to $26.5 \mathrm{~cm}$ ( $\log 16771$ ) with a mean of $19.3 \mathrm{~cm}$. In the Fe treatment, the range was from $9.2 \mathrm{~cm}$ (Kalamata) to $17.63 \mathrm{~cm}(72-5)$ with a mean of 12.6 (Table 1$)$. 
Table 1 Effect of $\mathrm{Fe}^{2+}$ stress on variables plant height, tiller number, leaf number and root length at the third weeks of Fe treatment

\begin{tabular}{|c|c|c|c|c|c|c|c|c|c|}
\hline \multirow[b]{2}{*}{ No. } & \multirow[b]{2}{*}{ Varieties } & \multicolumn{2}{|c|}{ Plant height (cm) } & \multicolumn{2}{|c|}{ Leaf number } & \multicolumn{2}{|c|}{ Tiller number } & \multicolumn{2}{|c|}{ Root length (cm) } \\
\hline & & Control & $\mathrm{Fe}^{2+}$ & Control & $\mathrm{Fe}^{2+}$ & control & $\mathbf{F e}^{2+}$ & Control & $\mathrm{Fe}^{2+}$ \\
\hline 1 & SAR05 & $47.5 a$ & $34.2 \mathrm{a}-\mathrm{c}$ & $10.7 a-d$ & $7.0 \mathrm{~d}$ & $1.1 \mathrm{a}-\mathrm{h}$ & $0.4 \mathrm{~b}-\mathrm{f}$ & $18.1 \mathrm{a}-\mathrm{c}$ & $12.1 \mathrm{a}-\mathrm{f}$ \\
\hline 2 & IR64 & $53.6 a-b$ & $36.1 \mathrm{a}-\mathrm{d}$ & 12.6de & $6.3 \mathrm{~cd}$ & $1.6 \mathrm{~g}-\mathrm{h}$ & $0.0 \mathrm{a}$ & $16.4 \mathrm{ab}$ & $10.1 \mathrm{ab}$ \\
\hline 3 & IR841 & 55.8a-c & $36.6 a-d$ & 10.9a-d & $6.6 \mathrm{~d}$ & $1.2 \mathrm{c}-\mathrm{h}$ & $0.5 f$ & $19.4 a-c$ & $12.1 \mathrm{a}-\mathrm{f}$ \\
\hline 4 & TXD88 & 53.3a-b & 36.7a-d & $12.5 \mathrm{de}$ & $6.0 \mathrm{~b}-\mathrm{d}$ & $1.2 \mathrm{~d}-\mathrm{h}$ & $0.0 \mathrm{a}$ & $20.4 a-d$ & $12.9 \mathrm{a}-\mathrm{g}$ \\
\hline 5 & SAHEL201 & $57.6 a-d$ & $37.5 \mathrm{a}-\mathrm{c}$ & $11.5 c-e$ & $6.4 \mathrm{~d}$ & $1.4 \mathrm{e}-\mathrm{h}$ & $0.1 \mathrm{a}-\mathrm{c}$ & $22 c-e$ & 11.8a-f \\
\hline 6 & YUNKENG & $52.9 \mathrm{a}-\mathrm{b}$ & $38.5 a-e$ & $7.2 \mathrm{a}-\mathrm{c}$ & $6.0 c-d$ & $0.0 \mathrm{a}$ & $0.2 \mathrm{a}-\mathrm{f}$ & $18.2 \mathrm{a}-\mathrm{c}$ & 13.3a-h \\
\hline 7 & NL23 & $53.8 \mathrm{a}-\mathrm{b}$ & $39.2 \mathrm{a}-\mathrm{f}$ & $7.6 a-c$ & $5.6 \mathrm{~b}-\mathrm{d}$ & $0.2 \mathrm{a}-\mathrm{d}$ & $0.1 \mathrm{a}-\mathrm{c}$ & $18.7 a-c$ & $12.4 \mathrm{a}-\mathrm{f}$ \\
\hline 8 & ARICA6 & $62.3 \mathrm{~b}-\mathrm{f}$ & 39.9a-f & $11.2 \mathrm{~b}-\mathrm{e}$ & $5.5 b-d$ & $0.8 a-h$ & $0.0 \mathrm{ab}$ & $16.5 a-b$ & $9.6 \mathrm{ab}$ \\
\hline 9 & ARICA7 & $54.8 \mathrm{a}-\mathrm{b}$ & $40.3 a-f$ & $11.3 c-e$ & $6.4 \mathrm{~d}$ & $1.3 \mathrm{~d}-\mathrm{h}$ & $0.0 \mathrm{ab}$ & $15.6 a$ & $12.2 \mathrm{a}-\mathrm{f}$ \\
\hline 10 & ARICA1 & $57 a-d$ & $41.0 \mathrm{a}-\mathrm{f}$ & $9.4 a-d$ & $7.1 \mathrm{~d}$ & $1.2 \mathrm{~b}-\mathrm{h}$ & $0.2 \mathrm{a}-\mathrm{f}$ & $15.2 \mathrm{a}$ & 11.0a-d \\
\hline 11 & ARICA2 & $63.1 \mathrm{~b}-\mathrm{f}$ & 41.6a-f & $7.8 \mathrm{a}-\mathrm{c}$ & $5.5 b-d$ & $0.2 \mathrm{a}-\mathrm{d}$ & $0.0 \mathrm{ab}$ & $15.2 \mathrm{a}$ & 12.9a-g \\
\hline 12 & ORYLUX4 & $64.6 \mathrm{~b}-\mathrm{f}$ & 42.9a-g & $15.2 \mathrm{~d}$ & $6.7 \mathrm{~d}$ & $1.9 \mathrm{~h}$ & $0.1 \mathrm{a}-\mathrm{c}$ & $17.7 \mathrm{a}-\mathrm{c}$ & $10.3 \mathrm{ab}$ \\
\hline 13 & WITA4 & 58.7a-e & $43.2 \mathrm{a}-\mathrm{h}$ & 9.5a-d & $6.4 \mathrm{~d}$ & $0.5 a-g$ & $0.2 \mathrm{a}-\mathrm{f}$ & $19.9 a-c$ & $14.2 \mathrm{~b}-\mathrm{n}$ \\
\hline 14 & YUNYINE & $62 b-f$ & 43.6a-h & $7.9 \mathrm{a}-\mathrm{c}$ & $5.2 \mathrm{~b}-\mathrm{d}$ & $0.0 \mathrm{a}$ & $0,0 a-d$ & $15.1 \mathrm{a}$ & $10.8 \mathrm{a}-\mathrm{d}$ \\
\hline 15 & ARICA3 & $63.7 b-f$ & 43.8a-h & $10.8 \mathrm{a}-\mathrm{d}$ & $5.2 \mathrm{~b}-\mathrm{d}$ & $1.1 \mathrm{~b}-\mathrm{h}$ & $0.1 \mathrm{a}-\mathrm{c}$ & 19.3a-c & $12.2 \mathrm{a}-\mathrm{f}$ \\
\hline 16 & JASMINE85 & $56.8 \mathrm{a}-\mathrm{d}$ & $45.4 \mathrm{~b}-\mathrm{h}$ & 9.6a-d & 7.0a-h & 0.8a-h & $0.5 \mathrm{f}$ & $15.0 \mathrm{a}$ & $10.4 \mathrm{ab}$ \\
\hline 17 & ARICA8 & $72.2 \mathrm{f}-\mathrm{h}$ & $49.2 b-i$ & $10.7 \mathrm{a}-\mathrm{d}$ & $6.2 c-d$ & $1.2 \mathrm{c}-\mathrm{h}$ & $0.0 \mathrm{a}-\mathrm{c}$ & $18.5 a-c$ & $11.2 \mathrm{a}-\mathrm{d}$ \\
\hline 18 & ORYLUX6 & $76.7 \mathrm{~g}-\mathrm{i}$ & $50.0 \mathrm{c}-\mathrm{i}$ & $10.7 a-d$ & $6.1 c-d$ & $0.9 a-h$ & $0.0 \mathrm{a}-\mathrm{d}$ & $18.5 a-c$ & $10.7 a-c$ \\
\hline 19 & CK73 & $68.6 \mathrm{~d}-\mathrm{g}$ & $50.8 \mathrm{a}$ & $6.5 a$ & $2.3 \mathrm{a}$ & $0.0 \mathrm{a}$ & $0.0 \mathrm{a}$ & $17.5 \mathrm{a}-\mathrm{c}$ & $11.5 a-d$ \\
\hline 20 & FOFIFA172 & $67.4 \mathrm{c}-\mathrm{g}$ & $50.9 c-j$ & $7.8 \mathrm{a}-\mathrm{c}$ & $5.5 b-c$ & $0.5 \mathrm{a}-\mathrm{f}$ & $0.0 a-d$ & $22.0 \mathrm{c}-\mathrm{e}$ & $15.7 \mathrm{e}-\mathrm{h}$ \\
\hline 21 & T0G16771 & $70.5 \mathrm{e}-\mathrm{g}$ & $51.8 c-j$ & $11.3 \mathrm{~b}-\mathrm{e}$ & $5.8 b-d$ & $1.5 \mathrm{f}-\mathrm{h}$ & $0.2 \mathrm{a}-\mathrm{f}$ & $26.5 u$ & $13 e-g$ \\
\hline 22 & $72-5$ & $67.7 \mathrm{c}-\mathrm{g}$ & $53.3 \mathrm{~d}-\mathrm{j}$ & $11.1 \mathrm{~b}-\mathrm{e}$ & $6.0 \mathrm{~b}-\mathrm{d}$ & $1 \mathrm{a}-\mathrm{h}$ & $0.0 \mathrm{ab}$ & $22.9 c-e$ & $17.6 \mathrm{~h}$ \\
\hline 23 & KALAMATA & $84.4 \mathrm{ij}$ & $55.8 \mathrm{e}-\mathrm{j}$ & $7.8 \mathrm{a}-\mathrm{c}$ & $5.8 b-d$ & $0.3 \mathrm{a}-\mathrm{e}$ & $0.1 \mathrm{a}-\mathrm{e}$ & $21.8 b-e$ & $9.2 \mathrm{a}$ \\
\hline 24 & SUAKOK08 & $82.1 \mathrm{~h}-\mathrm{j}$ & $56.5 f-j$ & 9.3a-c & $6.2 c-d$ & $0.6 \mathrm{a}-\mathrm{g}$ & $0.0 a-d$ & $20.1 \mathrm{a}-\mathrm{d}$ & $11.4 \mathrm{a}-\mathrm{e}$ \\
\hline 25 & NL19 & $86.1 \mathrm{ab}$ & $56.9 f-j$ & $8.3 a-d$ & $6.3 \mathrm{~cd}$ & $0.2 \mathrm{a}-\mathrm{d}$ & $0.0 \mathrm{a}-\mathrm{d}$ & $21.00-u$ & $15.0 \mathrm{c}-\mathrm{h}$ \\
\hline 26 & BOTRY & $85.7 \mathrm{i}-\mathrm{j}$ & $61.0 \mathrm{~g}-\mathrm{j}$ & $7.9 a-c$ & $2.2 \mathrm{a}$ & $0.4 a-f$ & $0.0 \mathrm{ab}$ & $21.3 \mathrm{~b}-\mathrm{d}$ & $13.1 \mathrm{a}-\mathrm{g}$ \\
\hline 27 & CK801 & $81.5 \mathrm{~h}-\mathrm{j}$ & $61.2 \mathrm{~g}-\mathrm{j}$ & $7.4 a-c$ & $6.2 c-d$ & $0.1 \mathrm{a}-\mathrm{c}$ & $0.0 a-d$ & $20.3 a-d$ & $16.9 \mathrm{gh}$ \\
\hline 28 & T0G6635 & $82.5 \mathrm{~h}-\mathrm{j}$ & $61.6 \mathrm{~h}-\mathrm{j}$ & $10.0 \mathrm{a}-\mathrm{d}$ & $6.2 c-d$ & $1.2 \mathrm{c}-\mathrm{h}$ & $0.0 \mathrm{ab}$ & $21.2 b-d$ & 16.1f-h \\
\hline 29 & SUPA & $90.3 j$ & $63.1 \mathrm{~b}-\mathrm{h}$ & $7.3 a-c$ & $4.4 \mathrm{bc}$ & $0.1 \mathrm{ab}$ & $0.0 \mathrm{a}$ & $21.2 \mathrm{~b}-\mathrm{d}$ & $9.3 a$ \\
\hline 30 & T0G6241 & 83.h-j & $65.06 \mathrm{~b}-\mathrm{i}$ & $9.8 \mathrm{a}-\mathrm{d}$ & $4.1 \mathrm{~b}$ & $0.9 a-h$ & $0.1 \mathrm{a}-\mathrm{c}$ & $25.2 \mathrm{de}$ & $15.9 e-h$ \\
\hline 31 & CK90 & $87.1 \mathrm{ij}$ & $68 . j$ & $6.8 \mathrm{ab}$ & $6.6 \mathrm{~d}$ & $0.00 \mathrm{a}$ & $0.0 \mathrm{a}-\mathrm{d}$ & 19.6a-c & $15.2 \mathrm{~d}-\mathrm{h}$ \\
\hline \multirow[t]{4}{*}{32} & SHAKA102 & $90.5 \mathrm{j}$ & $66.5 \mathrm{ij}$ & $8.4 a-d$ & 7.3a-i & $0.5 \mathrm{a}-\mathrm{f}$ & $0.4 \mathrm{~b}-\mathrm{f}$ & $18.9 a-c$ & 13.7a-h \\
\hline & Mean & 68.61 & 48.9 & 9.6 & 6.13 & 0.8 & 0.1 & 19.37 & 12.66 \\
\hline & SE & 7.1 & 10.80 & 2.55 & 1.12 & 0.64 & 0.24 & 3.11 & 2.61 \\
\hline & CV\% & 10.5 & 23.4 & 26.6 & 19.0 & 82.1 & 232.5 & 16.0 & 20.7 \\
\hline
\end{tabular}

Numbers followed by the same letter (s) in columns are not significantly different at $\mathrm{P} \leq 0.05$ using Duncan Multiple Range test

\subsection{Variation of dry shoot weight and root weight among the varieties and Fe treatments}

The study found that there were significant differences among the varieties. Shoot dry weight under control treatment ranged from $0.41 \mathrm{~g}$ (ARICA 7) to $1.5 \mathrm{~g}$ (NL 19) with a mean of $0.92 \mathrm{~g}$ while for Fe treatment shoot weight ranged from $0.12 \mathrm{~g}$ (CK 73) to $0.41 \mathrm{~g}$ (ARICA 8). On the other hand, root dry weight for control ranged from $0.06 \mathrm{~g}$ (ARICA 1 ) to 0.48 $\mathrm{g}$ (Orylux 4) with a mean of $0.26 \mathrm{~g}$ whereas, for Fe treatment, the range was from $0.04 \mathrm{~g}$ (CK 73) to 0.16 g (ARICA 8) with a mean of $0.10 \mathrm{~g}$ (Table 2). On average, $28.7 \%$ reduction was observed in plant height between control and Fe stress. This was the greatest reduction since leaf number was reduced by only $0.33 \%$, shoot weight by $71.7 \%$, root weight by $61 \%$ and root length decreased by $34.6 \%$. 
Table 2 Effect of $\mathrm{Fe}^{2+}$ on shoot dry weight and root dry weight of varieties exposed to control $\mathrm{Fe}$ and $300 \mathrm{ppm} \mathrm{Fe}^{2+}$ for three weeks

\begin{tabular}{|c|c|c|c|c|c|}
\hline \multirow[b]{2}{*}{ No. } & \multirow[b]{2}{*}{ Varieties } & \multicolumn{2}{|c|}{ Shoot dry weight (g) } & \multicolumn{2}{|c|}{ Root dry weight (g) } \\
\hline & & Control & $\mathbf{F e}^{2+}$ & Control & $\mathbf{F e}^{2+}$ \\
\hline 1 & SAR05 & $1.10 \mathrm{~b}-\mathrm{k}$ & $0.25 \mathrm{a}-\mathrm{c}$ & $0.45 \mathrm{~h}-\mathrm{k}$ & $0.09 \mathrm{ab}$ \\
\hline 2 & IR64 & $0.52 \mathrm{a}-\mathrm{b}$ & $0.19 \mathrm{ab}$ & $0.11 \mathrm{a}-\mathrm{c}$ & $0.07 \mathrm{ab}$ \\
\hline 3 & IR841 & $0.85 a-j$ & $0.25 a-c$ & $0.18 \mathrm{a}-\mathrm{f}$ & $0.08 \mathrm{ab}$ \\
\hline 4 & TXD88 & $1.06 \mathrm{a}-\mathrm{k}$ & $0.22 \mathrm{a}-\mathrm{c}$ & $0.21 \mathrm{a}-\mathrm{g}$ & $0.08 \mathrm{ab}$ \\
\hline 5 & SAHEL201 & $0.49 a-b$ & $0.19 \mathrm{ab}$ & $0.15 a-e$ & $0.09 \mathrm{ab}$ \\
\hline 6 & YUNKENG & $0.65 a-g$ & $0.22 \mathrm{a}-\mathrm{c}$ & $0.07 \mathrm{ab}$ & $0.08 \mathrm{ab}$ \\
\hline 7 & NL23 & $0.58 \mathrm{a}-\mathrm{f}$ & $0.15 a$ & $0.09 \mathrm{ab}$ & $0.06 \mathrm{a}$ \\
\hline 8 & ARICA6 & $0.96 \mathrm{a}-\mathrm{k}$ & $0.22 \mathrm{a}-\mathrm{c}$ & $0.16 a-f$ & $0.09 \mathrm{ab}$ \\
\hline 9 & ARICA7 & $0.41 \mathrm{a}$ & $0.21 \mathrm{a}-\mathrm{c}$ & $0.07 \mathrm{ab}$ & $0.12 \mathrm{a}-\mathrm{c}$ \\
\hline 10 & ARICA1 & $0.62 \mathrm{a}-\mathrm{g}$ & $0.26 a-c$ & $0.06 a$ & $0.08 \mathrm{ab}$ \\
\hline 11 & ARICA2 & $0.56 a-e$ & $0.25 a-c$ & $0.11 \mathrm{a}-\mathrm{c}$ & $0.09 \mathrm{ab}$ \\
\hline 12 & ORYLUX4 & $0.95 a-k$ & $0.23 a-c$ & $0.48 \mathrm{j}-\mathrm{k}$ & $0.07 \mathrm{ab}$ \\
\hline 13 & WITA4 & $0.65 a-g$ & $0.22 \mathrm{a}-\mathrm{c}$ & $0.13 a-d$ & $0.13 a-d$ \\
\hline 14 & YUNYINE & $0.44 a b$ & $0.16 \mathrm{ab}$ & $0.11 \mathrm{a}-\mathrm{c}$ & $0.05 a$ \\
\hline 15 & ARICA3 & $0.94 a-k$ & $0.21 \mathrm{a}-\mathrm{c}$ & $0.20 \mathrm{a}-\mathrm{f}$ & $0.08 \mathrm{ab}$ \\
\hline 16 & JASMINE85 & $0.72 \mathrm{a}-\mathrm{h}$ & $0.24 a-c$ & $0.17 \mathrm{z}-\mathrm{f}$ & $0.08 \mathrm{ab}$ \\
\hline 17 & ARICA8 & $1.51 \mathrm{jk}$ & $0.41 \mathrm{a}-\mathrm{f}$ & $0.36 \mathrm{e}-\mathrm{k}$ & $0.16 a-f$ \\
\hline 18 & ORYLUX6 & $1.15 \mathrm{c}-\mathrm{k}$ & $0.19 \mathrm{ab}$ & $0.23 a-h$ & $0.06 \mathrm{a}$ \\
\hline 19 & CK73 & $0.54 a-d$ & $0.12 \mathrm{a}$ & $0.11 \mathrm{a}-\mathrm{c}$ & $0.04 a$ \\
\hline 20 & F0FIFA172 & $0.92 \mathrm{a}-\mathrm{k}$ & $0.39 a-f$ & $0.15 a-e$ & $0.11 \mathrm{a}-\mathrm{c}$ \\
\hline 21 & T0G16771 & $1.23 \mathrm{f}-\mathrm{k}$ & $0.31 \mathrm{a}-\mathrm{e}$ & $0.44 \mathrm{~h}-\mathrm{k}$ & $0.13 z-d$ \\
\hline 22 & $72-5$ & $0.51 \mathrm{a}-\mathrm{c}$ & $0.29 a-d$ & $0.25 \mathrm{i}-\mathrm{a}$ & $0.11 \mathrm{ab}$ \\
\hline 23 & KALAMATA & $1.16 \mathrm{~d}-\mathrm{k}$ & $0.25 a-c$ & $0.24 a-h$ & $0.14 a-e$ \\
\hline 24 & SUAKOK08 & $0.69 a-h$ & $0.23 a-c$ & $0.19 a-f$ & $0.15 a-e$ \\
\hline 25 & NL19 & $1.56 \mathrm{k}$ & $0.28 \mathrm{a}-\mathrm{d}$ & $0.43 \mathrm{~g}-\mathrm{k}$ & $0.11 \mathrm{a}-\mathrm{c}$ \\
\hline 26 & BOTRY & $1.27 \mathrm{~g}-\mathrm{k}$ & $0.23 a-c$ & $0.33 c-j$ & $0.07 \mathrm{ab}$ \\
\hline 27 & CK801 & $1.32 \mathrm{~h}-\mathrm{k}$ & $0.36 \mathrm{a}-\mathrm{f}$ & $0.30-\mathrm{j}$ & $0.14 a-e$ \\
\hline 28 & T0G6635 & $1.22 \mathrm{e}-\mathrm{k}$ & $0.38 a-f$ & $0.47 \mathrm{i}-\mathrm{k}$ & $0.13 a-d$ \\
\hline 29 & SUPA & $1.09 \mathrm{~b}-\mathrm{k}$ & $0.28 \mathrm{a}-\mathrm{c}$ & $0.35 \mathrm{~d}-\mathrm{k}$ & $0.09 \mathrm{ab}$ \\
\hline 30 & T0G6241 & $0.80 \mathrm{a}-\mathrm{i}$ & $0.30 \mathrm{a}-\mathrm{d}$ & $0.55 \mathrm{k}$ & $0.12 \mathrm{a}-\mathrm{c}$ \\
\hline 31 & CK90 & $1.41 \mathrm{i}-\mathrm{k}$ & $0.28 \mathrm{a}-\mathrm{c}$ & $0.38 \mathrm{f}-\mathrm{k}$ & $0.12 a-b$ \\
\hline 32 & SHAKA102 & $1.35 \mathrm{~h}-\mathrm{k}$ & $0.27 a-c$ & $0.48 \mathrm{j}-\mathrm{k}$ & $0.11 \mathrm{a}-\mathrm{c}$ \\
\hline & Mean & 0.92 & 0.25 & 0.26 & 0.10 \\
\hline & $\begin{array}{l}\text { SE } \\
\text { CV\% }\end{array}$ & $\begin{array}{l}0.36 \\
41.9\end{array}$ & $\begin{array}{c}0.6 \\
24.8\end{array}$ & $\begin{array}{c}0.13 \\
74\end{array}$ & $\begin{array}{l}0.05 \\
52.8\end{array}$ \\
\hline
\end{tabular}

Numbers followed by the same letter (s) in columns are not significantly different at $\mathrm{P} \leq 0.05$ using Duncan Multiple Range test

\subsection{Variation of leaf bronzing among the varieties}

The first symptoms of Fe toxicity on the leaf were observed after ten days of Fe treatment (300 ppm Fe2+). However, varieties produced different degree of symptoms over the period. Leaf bronzing score after three weeks of Fe stress ranged from 1.8 (ARICA 1) to 6 (Suakok 8) with a mean of 3.6. Suakoko 8 which was used as one of the tolerant check showed a leaf bronzing score higher than the sensitive check IR64 (Table 3). 
Table 3 Iron toxicity symptom on rice genotypes after being exposed to $300 \mathrm{ppm} \mathrm{Fe}{ }^{2+}$ for three weeks

\begin{tabular}{|l|c|}
\hline Variety & Leaf bronzing score \\
\hline ARICA1 & 1.8 \\
\hline SAR05 & 2.2 \\
\hline ARICA7 & 2.3 \\
\hline CK801 & 2.5 \\
\hline SAHEL & 2.5 \\
\hline TXD88 & 2.5 \\
\hline JASMINE & 2.5 \\
\hline ARICA8 & 2.8 \\
\hline NL23 & 2.8 \\
\hline ORYLUX4 & 3.1 \\
\hline ORYLUX6 & 3.1 \\
\hline SHAKA201 & 3.1 \\
\hline ARICA6 & 3.2 \\
\hline IR64 & 3.3 \\
\hline YUNKENG & 3.3 \\
\hline YUNYINE & 3.5 \\
\hline CK90 & 3.5 \\
\hline TOG6635 & 3.5 \\
\hline TOG16771 & 3.5 \\
\hline IR841 & 3.6 \\
\hline ARICA3 & 3.7 \\
\hline ARICA2 & 3.8 \\
\hline WITA4 & 4.0 \\
\hline NL19 & 4.2 \\
\hline FOFIFA172 & 4.4 \\
\hline $72-5$ & 4.5 \\
\hline BOTRY & 4.9 \\
\hline KALAMATA & 5.0 \\
\hline SUPA & 5.1 \\
\hline TOG6241 & 5.5 \\
\hline CK73 & 5.9 \\
\hline SUAKOKO 8 & 6.0 \\
\hline Mean & 34.5 \\
\hline SE & \\
CV\% & \\
\hline
\end{tabular}

\subsection{Correlation of leaf bronzing with the other traits}

Correlation matrix of the traits revealed positive correlations between traits except with leaf bronzing which was negatively correlated with all the other traits. Plant height was significantly and positively correlated to shoot dry weight and root length while number of leaves was highly significantly correlated with number of tillers. Shoot, dry weight was significantly correlated with root length and root weight while root length was significantly correlated to root weight. Leaf bronzing was significantly correlated only with shoot dry weight (Table 4). It means that varieties with high leaf bronzing had small shoot biomass. 
Table 4 The correlation matrix Pearson between morphological traits of 32 varieties

\begin{tabular}{|l|c|l|l|l|l|l|l|}
\hline & $\begin{array}{l}\text { Plant } \\
\text { height }\end{array}$ & $\begin{array}{l}\text { Number of } \\
\text { Leaves }\end{array}$ & $\begin{array}{l}\text { Number of } \\
\text { Tillers }\end{array}$ & $\begin{array}{l}\text { Shoot dry } \\
\text { wt (g) }\end{array}$ & $\begin{array}{l}\text { Root Length } \\
\text { (cm) }\end{array}$ & $\begin{array}{l}\text { Root dry } \\
\text { wt (g) }\end{array}$ & $\begin{array}{l}\text { Leaf } \\
\text { bronzing }\end{array}$ \\
\hline Plant height & 1.000 & & & & & & \\
\hline Nb of leaves & 0.406 & 1.000 & & & & & \\
\hline Nb of Tillers & 0.197 & $0.848^{* *}$ & 1.000 & & & & \\
\hline Shoot wt (g) & $0.547^{*}$ & 0.449 & 0.348 & 1.000 & & & \\
\hline Root L (cm) & $0.600^{*}$ & 0.491 & 0.346 & $0.678^{*}$ & 1.000 & & \\
\hline Root wt(g) & 0.478 & 0.438 & 0.394 & $0.654^{*}$ & $0.577^{*}$ & 1.000 & \\
\hline Leaf bronzing & -0.402 & -0.487 & -0.407 & $-0.523^{*}$ & -0.478 & -0.345 & 1.000 \\
\hline
\end{tabular}

\subsection{Analysis of variance (ANOVA) for 32 rice varieties}

Analysis of variance was conducted to estimate the effects of variety (Var), Fe concentration and the interaction between variety and Fe concentration (Var x Fe Conc) on the variation of the different traits measured (Table 5). The results indicated that Fe concentration showed highly significant and very highly significant difference among the traits measured. Similarly very highly significant differences among varieties were revealed for all traits. On the other hand, non-significant differences were observed for the Fe concentration $\mathrm{x}$ variety interaction for plant height and root length. Tiller number and leaf bronzing showed very highly significant differences for the interaction while leaf number and shoot dry weight showed highly significant differences.

Table 5 Summary of ANOVA table showing variation in mean squares and F probability among varieties

\begin{tabular}{|l|c|c|c|c|l|l|l|l|}
\hline $\begin{array}{l}\text { Source of } \\
\text { variation }\end{array}$ & Df & $\begin{array}{l}\text { Plant } \\
\text { Height } \mathbf{( c m )}\end{array}$ & $\begin{array}{l}\text { Leaf } \\
\text { Number }\end{array}$ & $\begin{array}{l}\text { Tiller } \\
\text { Number }\end{array}$ & $\begin{array}{l}\text { Root } \\
\text { length } \\
\text { (cm) }\end{array}$ & $\begin{array}{l}\text { Root } \\
\text { Dry } \\
\text { Weight } \\
\text { (g) }\end{array}$ & $\begin{array}{l}\text { Shoot } \\
\text { Dry } \\
\text { Weight } \\
\text { (g) }\end{array}$ & Bronzing \\
\hline Rep & 3 & $766.25^{* * *}$ & $2.16 \mathrm{~ns}$ & $0.40 \mathrm{~ns}$ & $52.49^{* *}$ & $0.054^{*}$ & $1.78^{* * *}$ & $12.58^{* * *}$ \\
\hline Fe Conc. & 1 & $24391.79^{* * *}$ & $736.10^{* * *}$ & $28.05^{* * *}$ & $2846.69^{* * *}$ & $1.49^{* *}$ & $27.78^{* * *}$ & $833.49^{* * *}$ \\
\hline Var. & 31 & $1014.59^{* * *}$ & $9.71^{* * *}$ & $0.69^{* * *}$ & $3.28^{* * *}$ & $0.05^{* * *}$ & $0.29^{* * *}$ & $2.20^{* * *}$ \\
\hline Fe Conc. ${ }^{*}$ Var & 31 & $43.70 \mathrm{~ns}$ & $7.9^{* *}$ & $0.59^{* * *}$ & $1.17 \mathrm{~ns}$ & $0.03^{*}$ & $0 . .18^{* *}$ & $2.20^{* * *}$ \\
\hline Error (Var) & 3.73 & & & & & & & \\
\hline Total & 250 & & & & & & \\
\hline
\end{tabular}

\section{Discussion}

Excessive iron uptake has adverse effects on the vegetative growth of rice. It interferes with accumulation of dry matter by destruction of cells in the leaves due to the formation of reactive oxygen species (Tamuly et al., 2016). In this study, the effect of iron Fe2+ stress on all parameters was highly significant. Reduction of plant height, leaf and tiller number, shoot and root biomass and root length was observed over the three weeks of Fe stress, confirming that iron toxicity greatly affect rice plants by compromising their growth. This finding is consistent with previous studies, which showed that growth traits affected are number of tiller, shoot height, dry weight and decreased dry weight in varieties (Nyamangyoku et al., 2013). In addition, (Priyanga et al., 2012) recorded similar reductions for all traits. Growth reduction under Fe2+ treatment was observed in all the varieties tested but the range was variable. Some varieties showed lower reductions and fewer symptoms than others did. Overall, a significant variation was observed between the varieties tested concerning their relative performances under Fe toxicity stress. Leaf bronzing score is generally considered as an indicator of Fe toxicity stress level (Sikirou et al., 2015) and it has been often used to select rice varieties tolerant to Fe toxicity. In this study, eight varieties had a leaf bronzing score below 3: ARICA 1, Saro 5, ARICA 7, TXD88, Sahel201, CK801, NL23 and ARICA8 suggesting that they may have some tolerance to Fe toxicity. In terms of biomass accumulation under stress varieties ARICA8, FOFIFA172, TOG6635, CK801, TOG16771, T0G6241, 72-5, NL19, Shaka102, and CK90 had shoot and root biomass above the average. Taken together, CK801 and ARICA 8, which had 
both low leaf bronzing score and high biomass under stress, could be considered as tolerant to Fe toxicity in our experimental conditions. Surprisingly some of the tolerant checks (Suakoko 8 and WITA4) used in this experiment did not perform well. This is contrary to previous findings, which assert that in field and trials, these varieties combined high yield with iron toxicity tolerance (Sikirou et al., 2015). During the early stages of the experiment, it was observed that the roots of Suakoko 8 were quickly reaching the bottom of the trays while the other varieties' roots were elongating at a slower rate. It is suspected that the deep root character of Suakoko8, which in the field is beneficial, could have been detrimental in hydroponic conditions. Indeed, in field conditions, iron toxicity layer is within the first 2-15 cm and varieties that have short root systems are usually susceptible to iron toxicity while traditional varieties with deep root systems tend to be tolerant to Fe toxicity (Otoidobiga et al., 2015). In hydroponic culture, there is no possibility of escape or avoidance by going deeper.

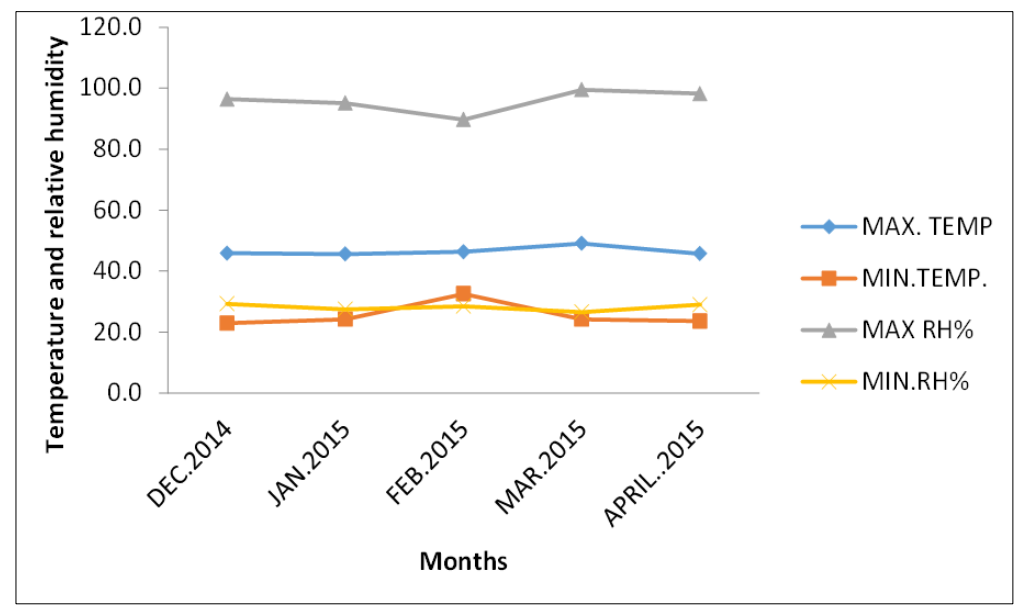

Figure 1 Maximum and minimum temperature and relative humidity during the experimental period

\section{Conclusion}

The present work helped in strengthening the background of genetic variation for Fe toxicity tolerance among varieties tested. It was observed, that varieties CK801 and ARICA8 were tolerant to iron toxicity as proven by their better performances under Fe toxicity stress. In addition, varieties CK801 and ARICA8 performed well in our study and helped to increase the rice ability in iron toxicity test. Moreover, molecular markers used in this study were also able to distinguish the different rice species.

\section{Compliance with ethical standards}

\section{Disclosure of conflict of interest}

No conflict of interest.

\section{References}

[1] Asch F, Becker M, Kpongor DS. A quick and efficient screen for resistance to iron toxicity in lowland rice. Journal of Plant Nutrition and Soil Science. 2005; 168(6): 764-773.

[2] Asch F, Becker M, Kpongor DS. A quick and efficent screen for resistance to iron toxicity in lowland rice Journal of plant Nutrition and soil Science. 2005; 168(6): 764-773.

[3] Bongoua-Devisme AJ, Cebron A, Kassin KE, Yoro GR, Mustin C, Berthelin J. Microbial communities involved in Fe reduction and mobility during soil organic matter (SOM) mineralization in two contrasted paddy soils. Geomicrobiology journal. 2013; 30(4): 347-361.

[4] Bongoua Devisme AJ, Cebron A, Kassin KE, Yoro GR, Mustin C, Betthelin J. Microbial communities involved in Fe reduction and mobility during soil organic matter (SOM) mineralization in two contrasted paddy soils. Geomicrobiology journal. 2013; 30(4): 347-361. 
[5] Dufey Inès et al. Construction of an integrated map through comparative studies allows the identification of candidate regions for resistance to ferrous iron toxicity in rice. Euphytica. 2015; 203(1): 59-69.

[6] Onaga, Geoffrey, Khady Nani. Dramé, Abdelbagi M. Isma. Understanding the regulation of iron nutrition. Functional plant biology. 2016; 43(8): 709-726.

[7] Gaikwad, Kiran B, et al. Yield-enhancing heterotic QTL transferred from wild species tocultivated rice Oryza sativa L. PloS one. 2014; 9(6).

[8] Brozynska, Marta, Agnelo Furtado, Robert J. Henry. Genomics of crop wild relatives. Expanding the gene pool for crop improvement. Plant biotechnology journal. 2016; 14(4): 1070-1085.

[9] Irri I. Standard evaluation system for rice. International Rice Research Institute, Philippine. 2002.

[10] Khadka N. Impact of Climate Change on Rice Yield Case. Nam Xong. 2016.

[11] Minhas PS, Obi Reddy GP. Edaphic Stresses and Agricultural Sustainability: An Indian Perspective. Agricultural Research. 2017; 1-14.

[12] Minhas, Paramjit Singh. Edaphic Stresses Concerns and Opportunities for Management. Abiotic Stress Management for Resilient Agriculture. Springer, Singapore. 2017; 73-94.

[13] Nyamangyoku IO, Bertin P . Mechanisms of Resistance to Ferrous Iron Toxicity in Cutivated Rices: Oryza sativa L., Oryza Glaberrima Steud and Interspecific Hybrids. International Journal of Agronomy and Plant Production. 2013; 4(10): 2570-2591.

[14] Nyamangyoku, I O, Bertin P. Mechanisms of Resistance to Ferrous Iron Toxicity in Cutivated Rices.Oryza sativa L., Oryza Glaberrima Steud and Interspecific Hybrids. International Journal of Agronomy and Plant Production. 2013; 4(10): 2570-2591.

[15] Otoidobiga $\mathrm{CH}$ et al. Dynamics and activity of iron-reducing bacterial populations in a West African rice paddy soil under subsurface drainage: Case study of kamboinse in burkina faso.Agricultural Sciences. 2015; 6(08): 860.

[16] Otoidobiga CH, et al. Dynamics and activity of iron-reducing bacterial populations in a West African rice paddy soil under subsurface drainage. Case study of kamboinse in Burkina Faso. Agricultural Sciences. 2015; 6(08): 860.

[17] Priya KC, Krishna, Terence S. Retp: Reliable event transmission protocol in a wireless sensor network. Emerging Trends in Computing, Communication and Nanotechnology (ICE-CCN). International Conference on. IEEE. 2013.

[18] Priya, KC, Krishna, Terence S. Retp: Reliable event transmission protocol in a wireless sensor network. Emerging Trends in Computing, Communication and Nanotechnology (ICE-CCN). International Conference on IEEE. 2013.

[19] Sikirou M, Saito K, Achigan-Dako EG, Dramé KN, Adam A, Venuprasad R. Genetic Improvement of Iron Toxicity Tolerance in Rice-Progress, Challenges and Prospects in West Africa. Plant Production Science. 2015; 1; 18(4): 423-34.

[20] Sikirou M, Saito K, Achigan-Dako EG, Dramé KN, Adam A, Venuprasad R. Genetic Improvement of Iron Toxicity Tolerance in Rice-Progress, Challenges and Prospects in West Africa. Plant Production Science. 2015; 1; 18(4): 423-34.

[21] Tamuly D. Bioremediation of iron toxicity in lowland rice ecosystem of Assam. Diss. AAU. 2016.

[22] Tetteh RN. Agricultural Sciences and Resources Management in the Tropics and Sub Tropics. Diss. RHEINISCHE FRIEDRICH-WILHELMS-UNIVERSITÄT BONN. Not quoted in the text of the paper. 2015.

[23] Tetteh RN. Chemical soil degradation as a result of contamination. A review Journal of Soil Science and Environmental Management. 2015; 6(11): 301-308.

[24] Venus et al. maintaining elevated Fe2+ concentration in solution culture for the development of a rapid and repeatable screening technique for iron toxicity tolerance in rice (Oryza sativa L.). Plant and soil. 2013; 372(12): 253-264.

[25] Venus, et al. maintaining elevated $\mathrm{Fe} 2+$ concentration in solution culture for the development of a rapid and repeatable screening technique for iron toxicity tolerance in rice (Oryza sativa L.). Plant and soil. 2013; 372(12): 253-264. 